\title{
Therapeutic action of tranexamic acid in hereditary haemorrhagic telangiectasia (HHT): Regulation of ALK-I/endoglin pathway in endothelial cells
}

\author{
Africa Fernandez-L.', Eva M. Garrido-Martin', Francisco Sanz-Rodriguez ${ }^{1,3}$, Jose-Ramon Ramirez ${ }^{4}$, \\ Carmelo Morales-Angulo², Roberto Zarrabeitia², Alfonso Perez-Molino², Carmelo Bernabéu', Luisa-María Botella' \\ 'Centro de Investigaciones Biológicas, CSIC, Ramiro de Maeztu, Madrid, Spain; ${ }^{2}$ Unidad de HHT, Hospital de Sierrallana, Torrelavega, \\ Santander, Spain; ${ }^{3}$ Facultad de Biología, Universidad Autónoma de Madrid, Madrid, Spain; ${ }^{4}$ Anatomia Patológica, Hospital Central de la \\ Defensa, Gomez Ulla, Spain
}

\begin{abstract}
Summary
Recurrent epistaxis is the most frequent clinical manifestation of hereditary haemorrhagic telangiectasia (HHT). Its treatment is difficult. Our objective was to assess the use of tranexamic acid (TA), an antifibrinolytic drug, for the treatment of epistaxis in HHT patients and to investigate in vitro the effects of TA over endoglin and ALK-I expression and activity in endothelial cells. A prospective study was carried out on patients with epistaxis treated with oral TA in the HHT Unit of Sierrallana Hospital (Cantabria, Spain). Primary cultures of endothelial cells were treated with TA to measure the levels of endoglin and ALK-I at the cell surface by flow cytometry. RNA levels were also measured by real-time $P C R$, and the transcriptional effects of TA on reporters for endoglin, ALK-I and the endoglin/ALK-I TGF-
\end{abstract}

\section{Keywords}

Endothelial cells, HHT, endoglin, ALK-I, tranexamic acid, fibrinolysis

\section{Introduction}

Hereditary haemorrhagic telangiectasia (HHT), or RenduOsler-Weber syndrome, is an autosomal dominant vascular disease with incomplete penetrance characterized by localized angiodysplasia. This is manifested as epistaxis, mucocutaneous and gastrointestinal telangiectases, and arteriovenous malformations in the pulmonary, cerebral or hepatic circulation (1). The prevalence is on average between one in 5,000 to one in 8,000 , although it is higher in some regions, such as the Jura region in France, Funen island in Denmark and certain Caribbean islands in the Netherland Antilles $(2,3)$. Its prevalence in Cantabria (Northern Spain) was calculated to be one in 12,000 in 1997 (4). However, according to more recent HHT Spanish beta pathway were assessed. The results showed that the fourteen HHT patients treated orally with TA improved, and the frequency and severity of their epistaxis were decreased. No complications derived from the treatment were observed. Cultured endothelial cells incubated with TA exhibited increased levels of endoglin and ALK-I at the protein and mRNA levels, enhanced TGF- $\beta$ signaling, and improved endothelial cell functions like tubulogenesis and migration. In summary, oral administration of TA proved beneficial for epistaxis treatment in selected patients with $\mathrm{HHT}$. In addition to its already reported antifibrinolytic effects, TA stimulates the expression of ALK-I and endoglin, as well as the activity of the ALK-I/endoglin pathway. population studies, in progress since 2003, the prevalence may be around one in 8,000 (Zarrabeitia et al., data not shown).

There are two main HHT types, type 1 and type 2 which are caused by mutations in endoglin and $A L K-1$ genes respectively $(5,6)$. In a few cases of the all HHT patients, around $2 \%$, the origin of the disease is a mutation in Smad4 gene leading to the combined syndrome of Juvenile Polyposis and HHT (7). Recently a third locus for an unknown HHT gene has been described in chromosome 5 (HHT3, [8]). All these genes are coding for proteins involved in the TGF $\beta$-signalling pathway, which is critical for the proper development of the blood vessels.

The most frequent clinical manifestation of HHT is epistaxis (nose bleeds), normally from light to moderate (9-11). However, some patients show severe epistaxis which notably interfere with 
their quality of life (12). The origin of this epistaxis is due to the existence of telangiectases on the nasal mucosa. These are focally dilated postcapilar venules, which in advanced phases show many layers of smooth muscle cells without elastic fibers, and very frequently directly connect with dilated arterioles (13). As a consequence of these vascular alterations, telangiectases are very sensitive to slight traumata and even to the friction with the air when breathing, which gives rise to nose bleeds.

There is no optimal treatment for the epistaxis in HHT patients. Many different therapies have been assayed, but none of them with conclusive results (14-17). The use of antifibrinolytic agents for the treatment of HHT patients, systemically administered (intravenously, intraparenteral, orally) was described for the first time by Saba et al. (18), using oral administration of aminocaproic acid to two patients. The result was satisfactory with an improvement in epistaxis and the associated anemia. More recently, other authors have also reported good results using tranexamic acid (TA), both orally and topically $(20,21)$. TA may also be administered endovenously in cases of very acute bleeding episodes (10). TA is a derivative of the amino acid lysine, 4 (aminomethyl cyclohexanecarboxyl) (22). It binds reversibly to plasminogen, avoiding fibrin degradation by plasmin $(23,24)$. TA enters into the extravascular space and accumulates in the tissues (25). The basis for its efficiency relies on the inhibition of the fibrinolytic activity in the tissues that leads to clot stabilization. TA or other antifibrinolytic lysineanalogues are indicated in hereditary deficiencies of the plasmin/ PAI-1 system, in acute episodes of bleeding in von Willebrand disease or acute hemophilia, but in the latter cases the diseases can be treated supplying the corresponding deficient coagulation factor. TA is also indicated in severe bleedings like after surgery and in gynecologic bleedings like menorrhagia and in hyperfibrinolysis. The latter is exactly the case of HHT bleeding showing hyperfibrinolysis secondary to intravascular coagulation (22). The presence of local hyperfibrinolysis with increase in t-PA justifies the use of an antifibrinolytic agent, such as TA for the management of HHT epistaxis. By contrast, other hemostatic drugs increasing the release of coagulant factors, as desmopresin, are more indicated in the management of other hemorrhagic hereditary diseases as hemophilia or von Willebrand disease.

TA is not yet broadly used to treat HHT since there are few reports on the benefits of its oral, topical or endovenous administration in HHT. The scarce studies are either case reports, or include at the most three patients. In the present study we report the efficiency of orally administered TA in 14 HHT patients with moderate or severe epistaxis, according to the experience of the Spanish HHT reference unit. The aim of this study was to assess TA in in-vitro experiments with cultured endothelial cells, quantifying endoglin and ALK-1, deficient in HHT patients, to understand at molecular level why TA may be especially useful in HHT. These results are implemented with clinical data showing an improvement in epistaxis. These data do not represent a clinical trial because it is almost impossible to do a clinical trial in a rare disease, where the treatment is required to stop bleeding, and hence is hard to leave patients without treatment (controls). However, the comparison of bleeding parameters before and after the treatment is in itself a kind of control. Both, the in-vitro results and the success with patients makes TA a encouraging drug to improve the quality of life in HHT.

\section{Material and methods}

\section{Clinical study of HHT patients}

A prospective study of HHT patients attending the HHT unit of Sierrallana hospital between 2001 and the first semester of 2006, was conducted. HHT patients were diagnosed according to $\mathrm{Cu}-$ raçao criteria (26). Those patients with moderate or severe epistaxis which interfered with their quality of life were treated with TA when they did not show any contraindication. The treatment normally consisted of $500 \mathrm{mg}$ oral TA (Amchafibrin, Fides Ecopharma, Rota-Research group) every eight hours, with corrections according to renal function. Doses of TA in some cases may reach up to $1 \mathrm{~g}$ every eight hours. Before the study, all patients were subjected to blood tests including hemogram, plasma biochemical studies, coagulation studies and genetic studies to look for the mutation origin of the disease. Moreover, a complete screening of head and neck was made by an ear-nose-throat specialist involved in the screening of HHT patients. The efficiency of the TA treatment was evaluated according to the transfusion needs, and the frequency and intensity of epistaxes following (27). To measure the degree of satisfaction a scale from I (very satisfied) to IV (very unsatisfied) was used.

\section{Cell culture}

BOECs (blood outgrowth endothelial cells) and HUVECs (human umbilical vein endothelial cells) were obtained as described (28). HMECs (human microvasculature endothelial cells) were incubated in the same endothelial enriched medium used for BOECs and HUVECs as described (29). Cells were incubated in the presence or absence of $2 \mathrm{mM}$ TA diluted directly into the culture medium. $\varepsilon$-amino caproic acid (AC), another antifibrinolytic drug, was used for comparison purposes with TA (both chemicals from Fides Ecopharma, Rotta-Research group). The plasmin protease inhibitor, Aprotinin (AP) from Sigma-Aldrich, was also used as an agent mimicking the action of TA and AC. All the assays were made in the presence of $10 \%$ FBS supplemented medium, likely to contain around 1.4 and $1.5 \mathrm{U} / \mathrm{ml}$ of plasminogen biological activity measured by radioimmunodiffusion (The Binding Site LTD, Birmingham, UK)

\section{Flow cytometry analysis}

Measurements of endoglin, ALK-1 and PECAM (CD31) levels on the cell surface were carried out by flow-cytometric analysis from HUVECs and BOECs using monoclonal antibodies P4A4, anti-ALK-1 (R\&D Systems) and HC1/16, respectively, according to the procedure described by Fernandez-L. et al. (28) and Sanz-Rodriguez et al. (29).

\section{Real-time PCR}

Endoglin and ALK-1 oligonucleotides were purchased from Sigma and chosen according to Roche's software for real-time PCR. Total RNA was extracted from endothelial cells (HUVECs, BOECs, and HMECs) using the RNAeasy kit (Qiagen), retrotranscribed using the AMV RT kit from Roche, and amplified in a real time PCR using the Universal Human Probe Roche library and the Real-time PCR kit from Roche.

The assays were performed in triplicates, compared with two different types of endogenous controls (18S rRNA and GAPDH) 
and repeated at least twice. The oligonucleotide sequences and probe numbers for endoglin, ALK-1, GAPDH and 18S RNA used in the PCR were:

- Endoglin left oligo: 5' AGCCTCAGCCCCACAAGT 3'; right oligo: 5' GTCACCTCGTCCCTCTCG 3'

- Human 41 Probe of Roche library for endoglin

- ALK-1/ACVRL1 left oligo: 5' ATCTGAGCAGGGCGACAC 3'; right oligo 5' ACTCCCTGTGGTGCAGTCA 3'

- Human 79 probe of Roche library for ALK-1/ACVRL1

- GAPDH left oligo: 5' AGCCACATCGCTCAGACAC 3'; right oligo: 5' GCCAATACGACCAAATCC 3'

- Human 60 probe of Roche library for GAPDH

- 18S rRNA left oligo: 5' CTCAACACGGGAAACCTCAC 3'; right oligo: 5' CGCTCCACCAACTAAGAACG 3'

- Human 77 probe of Roche library for 18S rRNA.

\section{Wound healing and tube formation assays}

In vitro scratched wounds were created by scraping confluent HMEC monolayers in P-24 plate wells with sterile disposable pipet tips. The remaining cells were washed with Hanks Buffered Salt Solutions (HBSS) buffer (Hanks, Gibco) and incubated with Endolthelial Basic Medium (EBM)-2/Endothelial Growth Medium (EGM)-2 medium (Clonetics, Cambrex) in the absence or presence of antifibrinolytic drugs, TA, AC and AP, up to 24 hours (h). Endothelial cell migration into denuded area was monitored by photography of the plates at different times.

For tube formation assays, HMECs were plated in EBM/ EGM-2 culture medium in the absence or presence of antifibrinolytic drugs, TA, AC and AP on P-6 well matrigel plates (Becton Dickinson) and incubated at $37^{\circ} \mathrm{C}$, as indicated by the manufacturer. Tube formation was monitored for $24 \mathrm{~h}$.

\section{Cell transfection and reporter assays}

Transient transfections of BOECs were made using Superfect (Qiagen) with reporters for endoglin promoter: pCD105

Table I: Characteristics of the HHT patients.

\begin{tabular}{l|l|l}
\hline No/age/sex & Mutation & Previous treatments \\
\hline I/47/M & Not found & None \\
\hline 2/80/M & ENG/HHTI & Cauterization, A/P package \\
\hline $3 / 69 / \mathrm{M}$ & ALKI/HHT2 & Cauterization, A/P package \\
\hline $4 / 42 / \mathrm{M}$ & ALKI/HHT2 & None \\
\hline $5 / 49 / \mathrm{F}$ & ALKI/HHT2 & Cauterization, A/P package \\
\hline $6 / 47 / \mathrm{F}$ & ALKI/HHT2 & None \\
\hline $7 / 59 / \mathrm{M}$ & ENG/HHTI & Cauterization, A/P package \\
\hline $8 / 45 / \mathrm{F}$ & ALKI/HHT2 & None \\
\hline $9 / 78 / \mathrm{F}$ & ALKI/HHT2 & None \\
\hline I0/4I/F & ALKI/HHT2 & None \\
\hline II/49/F & ALKI/HHT2 & None \\
\hline I2/48/F & ENG/HHTI & Cauterization, A/P package \\
\hline I3/43/M & Not found & A/P package \\
\hline I4/50/F & ALKI/HHT2 & None \\
\hline M, male; F, female; A/P, anterior/posterior. &
\end{tabular}

$(-450 /+350)$ in pXP2 $(30,31)$, here denoted as pENG/pXP2 and ALK-1 promoter: pALK-1/pGL2, a construction encompassing the first exon and around 1.3-kb upstream of the ALK-1 genomic DNA inserted in Sac I and Xho I sites of pGL2 polylinker. The TGF $\beta$ pathway reporters were 3TP-lux, ALK-5 responsive $(\mathrm{CAGA})_{12}$ Luc, or ALK-1 responsive (BRE) $)_{2}$-Luc (32). Reporter vectors were co-transfected or not with expression vectors coding for a dominant negative mutant (ALK-1-K229R), or a constitutively active mutant (ALK-1-Q201D) of ALK-1. Relative luciferase units (RLU) were measured in a TD20/20 luminometer (Promega, Madison, WI, USA).

Samples were co-transfected with the SV40- $\beta$ galactosidase expression vector to correct for transfection efficiency. Measures of $\beta$-galactosidase activity were performed using Galacto-light (Tropix). The transfections were made in triplicates and repeated at least in three independent experiments. Representative experiments are shown in the figures.

\section{Statistics}

All data presented represent mean $\pm \mathrm{SD}$. Differences in mean values were analyzed using Student's t-test. P-values $<0.05$ were considered to be statistically significant. In the figures, the statistically significant values are marked with asterisks as follows: $* \mathrm{p}<0.05,{ }^{*} \mathrm{p}<0.01,{ }^{* * *} \mathrm{p}<0.001$.

\section{Results}

\section{Clinical treatment with TA of a group of Spanish HHT patients}

Previous reports on the successful use of TA for nose bleed management are based on one or, at the most, three patients $(20,21$, 33). This fact led us to follow up the clinical use of oral TA treatment in a larger series of Spanish HHT patients. In parallel, invitro research was conducted in the laboratory to assess TA effects at cellular and molecular levels.

The HHT unit of Sierrallana Hospital (Cantabria, Spain) has screened from 2003 up to now, more than 150 patients belonging to around 40 different families. Of these, a total of 14 patients which had severe epistaxis interfering with their quality of life volunteered for a treatment with oral TA. In all these cases side effect risks of thrombosis were absent. The type of patient, mutation, sex, and management of the epistaxis before the treatment are recorded in Table 1. The TA treatment and the severity of epistaxis before and after the treatment are shown in Table 2. All patients showed a decrease in the intensity and frequency of nose bleeds after the first week of treatment, and when the treatment was discontinued the amount of haemorrhages increased immediately. At this moment, all of them continue with the TA treatment and none has presented adverse side effects derived from it until now.

\section{Effect of TA on the amount of ALK-I and endoglin at the surface of endothelial cells}

As HHT1 and 2 are caused by endoglin and ALK-1 haploinsufficiency, respectively, we have explored the in-vitro TA effects on these two genes at the cellular level.

First, we decided to investigate if TA in a range of concentrations (from 0 to $10 \mathrm{mM}$ ) was having any effect on the amount 
Table 2: Tranexamic acid treatment and its efficiency.

\begin{tabular}{|c|c|c|c|c|c|c|c|}
\hline No & Dose/type & $\begin{array}{l}\text { Period of } \\
\text { treatment }\end{array}$ & $\begin{array}{l}\text { Previous } \\
\text { transfusions }\end{array}$ & $\begin{array}{l}\text { Posttreatment } \\
\text { transfusions }\end{array}$ & $\begin{array}{l}\text { Pretreatment } \\
\text { epistaxis (I/F)* }\end{array}$ & $\begin{array}{l}\text { Posttreatment } \\
\text { epistaxis (I/F)* }\end{array}$ & Satisfaction \\
\hline 1 & $500 \mathrm{mg} / \mathrm{/} 2 \mathrm{~h}$ & $2 m$ & No & No & $\| I I / I I$ & $1 / I$ & II \\
\hline 2 & $500 \mathrm{mg} / 8 \mathrm{~h}$ & $24 \mathrm{~m}$ & $>5$ & $<5$ & III/IIII & $\|/\|$ & II \\
\hline 3 & $500 \mathrm{mg} / 8 \mathrm{~h}$ & $25 \mathrm{~m}$ & $<5$ & No & III/IIII & $1 / 1$ & II \\
\hline 4 & $500 \mathrm{mg} / 8 \mathrm{~h}$ & $2 m$ & No & No & $\|/ /\|$ & $1 / 1$ & II \\
\hline 5 & $1000 \mathrm{mg} / 8 \mathrm{~h}$ & $16 \mathrm{~m}$ & $<5$ & $<5$ & $I I I / I I$ & $\| / / I$ & III \\
\hline 6 & $500 \mathrm{mg} / 8 \mathrm{~h}$ & $\mathrm{II} \mathrm{m}$ & $<5$ & No & $\| I I / I I$ & $1 / 1$ & ॥ \\
\hline 7 & $500 \mathrm{mg} / 8 \mathrm{~h}$ & $12 \mathrm{~m}$ & $<5$ & $<5$ & III/IIII & $11 / 11$ & III \\
\hline 8 & $500 \mathrm{mg} / \mathrm{/2} \mathrm{h}$ & $4 \mathrm{~m}$ & No & No & $\| / / I$ & $1 / 1$ & I \\
\hline 9 & $500 \mathrm{mg} / / 2 \mathrm{~h}$ & $6 \mathrm{~m}$ & No & No & $\|1 /\|$ & $1 / 1$ & II \\
\hline 10 & $500 \mathrm{mg} / 8 \mathrm{~h}$ & $4 \mathrm{~m}$ & No & No & $11 / / I$ & $1 / 1$ & II \\
\hline II & $500 \mathrm{mg} / 8 \mathrm{~h}$ & $4 \mathrm{~m}$ & No & No & $11 / 11$ & $1 / 1$ & II \\
\hline 12 & $500 \mathrm{mg} / 8 \mathrm{~h}$ & $8 \mathrm{~m}$ & No & No & III/IIII & $11 / I I$ & II \\
\hline 13 & $1000 \mathrm{mg} / 8 \mathrm{~h}$ & $5 \mathrm{~m}$ & No & No & III/II & $11 / 1$ & II \\
\hline 14 & $1000 \mathrm{mg} / 8 \mathrm{~h}$ & $12 \mathrm{~m}$ & No & No & $\| / / I$ & $\mathrm{I} / \mathrm{I}$ & II \\
\hline
\end{tabular}

of ALK-1 and endoglin at the surface of endothelial cells (Fig. 1A). The effect on the endothelial marker PECAM was used as a control, and an irrelevant antibody was taken as negative control. The amount of cell-surface endoglin was increased 3-4 times, even at the lowest concentration $(0.5 \mathrm{mM})$. In another set of experiments, at $2 \mathrm{mM} \mathrm{TA}$ (since it yields good response), the amount of endoglin and ALK-1 increased by $1.5-$ and 1.75 -fold, respectively (Fig. 1B). In Figure 1C, the endoglin and ALK-1 induction after $2 \mathrm{mM}$ TA in BOECs (other type of primary endothelial cells) (28) is shown. The upregulation of endoglin and ALK-1 by TA treatment was also explored after AC treatment and the plasmin protease inhibitor AP, in a dose-response experiment, to investigate if other antifibrinolytic agents were behaving similarly. In this case the endothelial established cell line HMEC was used for purposes of homogeneity of results, and they were grown on Dubbecco's Modified Eagle Medium (DMEM). This cell line has lower basal levels of endoglin which allowed us to observe stronger induction changes after treatments than in primary cultures. As can be seen in Figure 1D and $E$ all of them seem to increase the protein levels of endoglin and ALK-1, with TA being the most efficient in increasing endoglin levels at the concentrations used. TA and AP were equally efficient stimulating protein levels of ALK-1 on the cell surface. These experiments show that antifibrinolytic drugs, TA, AC and AP increase the levels of endoglin and ALK-1 on the endothelial cell surface, but the mechanism involved may be either posttranscriptional stabilization of these surface proteins, induction at transcriptional level or both mechanisms at the same time. To discriminate among these possibilities, the effects of TA, AC, and AP on endoglin and ALK-1 RNA levels were investigated. To this end, HMECs were treated with TA, AC $(2 \mathrm{mM})$ and AP $(5 \mu \mathrm{g} / \mathrm{ml})$. These single doses were selected as the most convenient leading to significant protein increase, and good cell viabil- ity after the results shown in Figure 1D and E. As shown in Figure $2 \mathrm{~A}$, the levels of endoglin and ALK-1 RNA are significantly induced after treatments. However, the most efficient induction is achieved in the case of TA, with the effect of AC and AP induction on endoglin and ALK-1 RNA levels being very weak and not significant. Since TA doubles endoglin/ALK-1 mRNA levels while the corresponding protein levels are induced by $10-$ to 14-fold, at this point we must argue that TA is affecting both, transcriptional and post-transcriptional processes, while the effects of AC and AP at the RNA level may be too weak to be observed after $24 \mathrm{~h}$. Since TA has significant affects at endoglin and $A L K-1$ RNA levels, the transcriptional effects will be only explored after TA treatment.

Thus, the effect of TA was assessed at the transcriptional level on endoglin and promoters. Figure 3A shows the results of transfecting endothelial cells (BOECs) with constructs representing endoglin and $A L K-1$ promoters. In both cases, the TA treatment doubles the transcription rate of endoglin and $A L K-1$ promoter constructs.

As published previously (28), ALK-1 affects endoglin promoter activity. Accordingly, using a dominant negative ALK-1 form (ALK-1-KR), endoglin promoter activity is blocked, while a constitutively active ALK-1 mutant (ALK-1-QD) increases endoglin promoter activity (Fig. 3A). In both cases TA treatment does not affect this response which argues in favour of TA acting, either directly or indirectly, through the TGF- $\beta$ pathway.

\section{Effect of TA on the TGF- $\beta$, ALK-5 and ALK-I/endoglin pathways}

Since TA enhances endoglin and ALK-1 expression, at protein, RNA and promoter levels, we decided to ascertain if TA also has an effect on the TGF- $\beta$ pathways active in endothelial cells, ALK-5 and ALK-1/endoglin (32, 34). A general TGF- $\beta$ reporter 


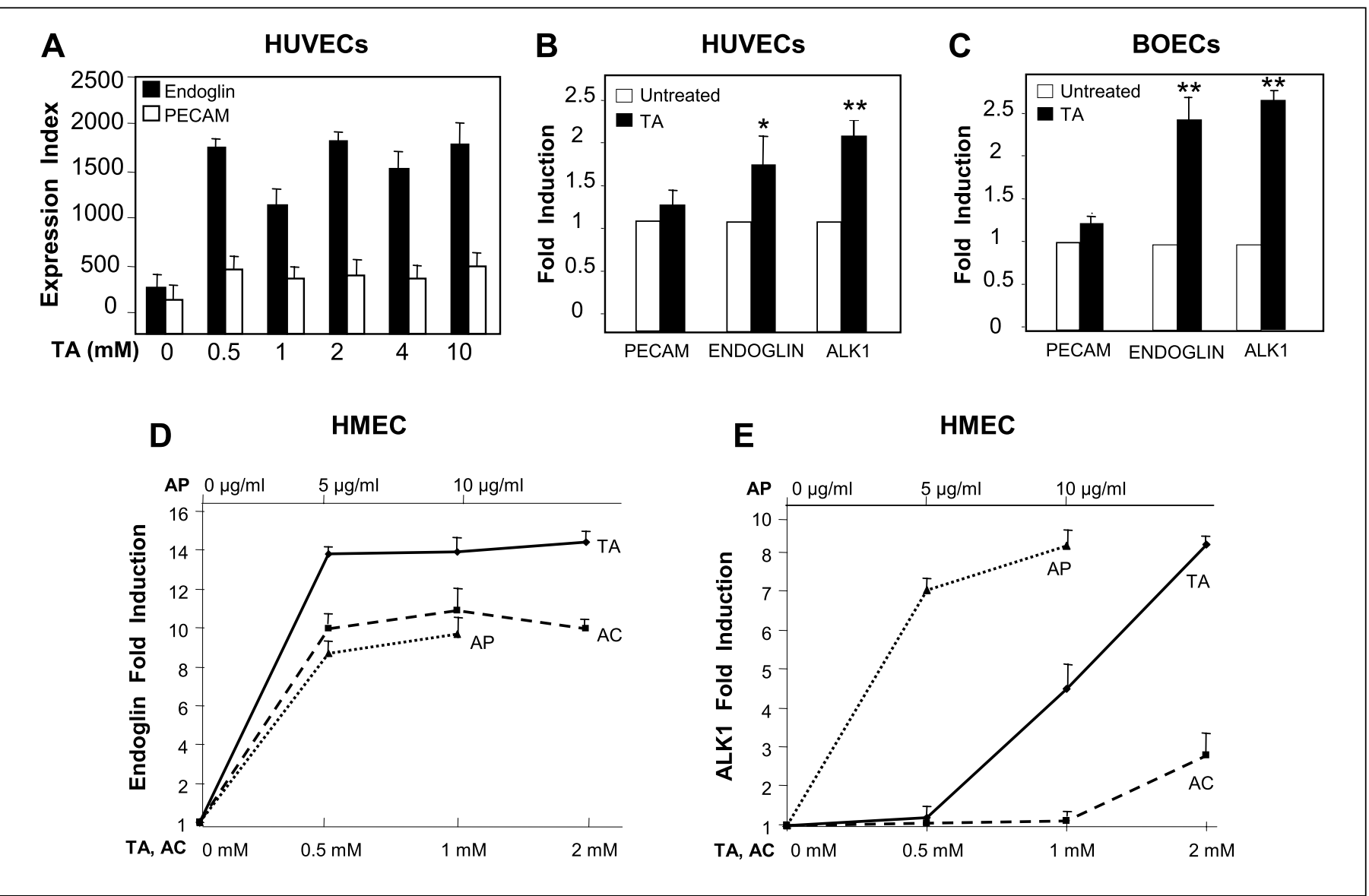

Figure I: Effect of TA on the surface expression of endoglin and ALK-I in endothelial cells. A) HUVECs were cultured in the absence or presence of increasing concentrations of TA $(0.5 \mathrm{mM}$ to $10 \mathrm{mM}$ ) for $24 \mathrm{~h}$. Endoglin and PECAM levels were measured by flow $\mathrm{c}$ ytometry. B) HUVECs were incubated in the absence or in the presence of 2 mMTA and the levels of endoglin, ALK-I and PECAM were measured after $24 \mathrm{~h}$ and compared with untreated cells. The results of the flow cytometry are expressed in fold induction levels. ${ }^{*} \mathrm{P}<0.05$; **P $<0.0 \mathrm{I}$. C) BOECs were treated with $2 \mathrm{mMTA}$, and the levels of endoglin, ALK-I, and PECAM were measured after $24 \mathrm{~h}$. The results

derived from the PAI-1 promoter, 3TP-lux, an ALK5-pathway specific reporter, (CAGA) ${ }_{12}$-luc, and the ALK-1/endoglin-pathway specific reporter, BRE-luc, were used. The 3TP-lux and the (BRE) $)_{2}$-luc reporters showed a significant increase of their activity following TA treatment, but, while 3TP-lux activity was slightly increased ( 1.5-fold), (BRE) $)_{2}$-luc reporter was strongly stimulated ( 4-fold). The (CAGA) 12 -luc reporter did not show any change after TA treatment. Since 3TP-lux contains PAI-1 proximal promoter encompassing AP1 and other transcription factor sites in addition to the ALK-5/Smad3 responsive element, the 1.5-fold increase of the 3TP-lux reporter by TA may be the consequence of other trancription factor(s) induced by TA, and it is interesting in itself due to the PAI-1 antifibrinolytic function.

In summary, TA seems to specifically stimulate the TGF $\beta /$ ALK-1/endoglin pathway, though we do not know the mechanism yet. of flow cytometry are expressed in fold induction with respect the untreated control. D and E) Endoglin (D) and ALK-I (E) content was measured by flow cytometry in endothelial cells untreated or treated with different amounts of TA, AC, and AP, as indicated. The dose of $20 \mu \mathrm{g} / \mathrm{ml}$ AP was also tested, but the survival of cells was significantly reduced, and therefore the corresponding data is not included in the figure. The results of the flow cytometry are normalized with PECAM expression and expressed in fold induction with respect the untreated control. Experiments were made in triplicates, repeated at least twice, and a representative experiment in each panel is shown.

\section{Plasminogen inhibitors favour endothelial cell functions depending on the ALK-I/endoglin pathway: cord formation and migration}

We have just shown that TA is specifically stimulating the TGF $\beta$ / ALK-1/endoglin pathway, without affecting TGF $3 /$ ALK5 signalling. Therefore we performed two types of functional experiments under control of this pathway according to literature $(32,34)$ : cord formation (angiogenesis) and cell migration after wound healing.

Cord formation, is an in-vitro experiment mimicking the angiogenic function of endothelial cells. Figure 4 shows cord formation in a time-course experiment. As can be seen, tubes develop faster in TA-treated endothelial cells. After six hours we can observe that the cord network is completely developed in TA treated cells; this happens around eight hours in AC and AP treatment and only after $12 \mathrm{~h}$ in untreated controls.

TA, AC and AP promote a faster migration of cells, so that an artificial monolayer disruption is closed between 15 and $24 \mathrm{~h}$. In 


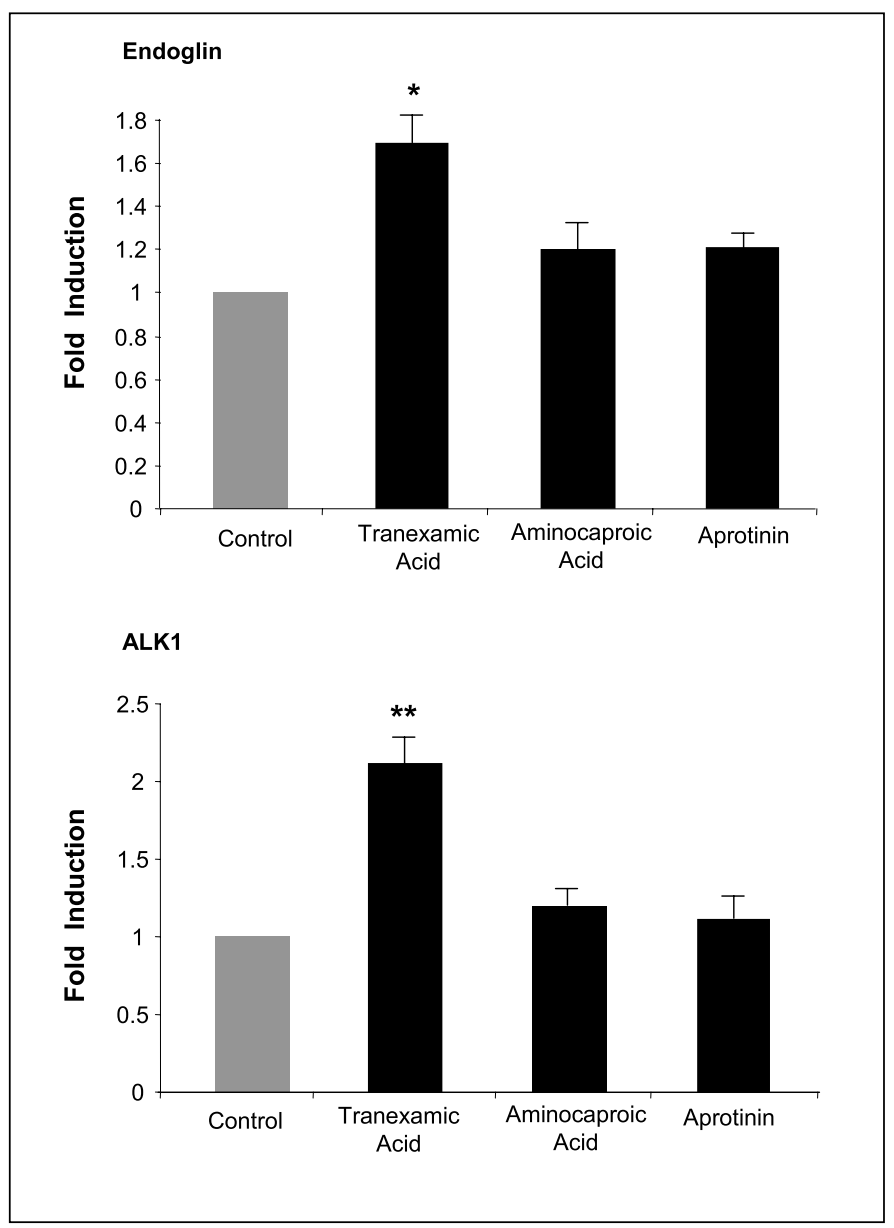

Figure 2: Effect of TA on endoglin and ALK-I transcription. Endothelial cells were treated for $24 \mathrm{~h}$ with TA $(2 \mathrm{mM}), A C(2 \mathrm{mM})$ and AP $(5 \mu \mathrm{g} / \mathrm{ml})$. For real-time PCR, total RNA was extracted, retro-transcribed and amplified. Quantification of the number of ALK-I and endoglin RNA molecules was carried out and compared to endogenous controls of I8S ribosomal RNA and GAPDH. The experiment was made in triplicates, and repeated for the three types of endothelial cells. A representative experiment of HMECs is shown, although similar results were obtained with HUVECs and BOECs.

untreated cells (controls), some remaining layer discontinuity is still observed after $24 \mathrm{~h}$. TA treatment is the strongest in stimulating cell migration with a closure around $18 \mathrm{~h}$ followed by $\mathrm{AC}$ and AP closing the wound at $24 \mathrm{~h}$ (Fig. 5).

These two functional experiments support the involvement of TA action in triggering the TGF $\beta /$ ALK-1/endoglin pathway in endothelial cells. On the other hand, the experiments suggest that TA way of action is indirect through plaminogen activation inhibition, since treatment with AC and AP, both inhibitors of this activation, leads to similar results.

\section{Discussion}

Epistaxis is the most common symptom in HHT patients, appearing in $90 \%$ of them. Epistaxis shows up before the third decade of age, but patients normally worsen with age, and epistaxis becomes very severe in $18 \%$ of the cases leading to anemia as

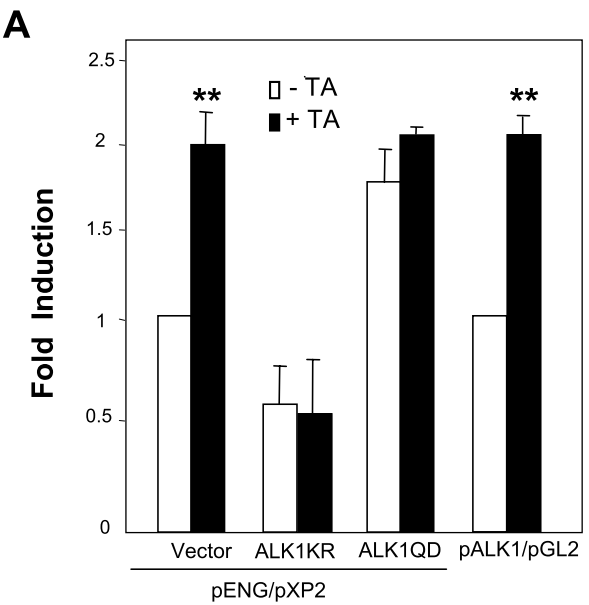

B



Figure 3: Effect of TA on the TGF $\beta$ pathways in endothelial cells. A) Effect of TA on endoglin and ALK-I promoter activity. BOECs were transiently transfected with a construction of endoglin promoter, $\mathrm{pENG} / \mathrm{pXP2}$, and a construct of ALK-I promoter in pGL2. Kinase dead ALK-I (ALK-I-KR), constitutively active ALK-I (ALK-I-QD) or empty expression vector ( $p c D N A 3$ ) were cotransfected, as indicated. Luciferase activity was measured in 2 mMTA-treated cells and expressed as fold induction with respect the untreated cells. B) BOECs were transiently transfected with different reporters for the TGF $\beta$ pathway, the general TGF $\beta$ responsive 3TP-lux, the ALK- 5 specific CAGA-luc, and the ALK-I/endoglin-specific BRE-luc. Transfected cells were incubated in the presence or in the absence of 2 mMTA. Reporter activity is expressed in relative luciferase units. All the experiments were made by triplicate and repeated at least three times. The results shown are a representative experiment.

well as to a decrease in the patient's life quality (12). Different treatments have been assayed in the past including cauterization, estrogen/progesterone treatment, the injection of sclerosing agents (i.e. ethyblock) in the telangiectases, septodermoplastia, embolization or radiotherapy, all of them with a limited and temporal efficiency $(33,35,36)$. After all these treatments, an increase in the fibrinolytic activity of the telangiectasic tissue is later observed (37). In all cases, this activity was mediated by a plasminogen activator increase.

From these observations, several authors began the use of antifibrinolytic drugs such as $\varepsilon$-aminocaproic acid initially, and TA later for the treatment of HHT patients (18-21). TA is an 

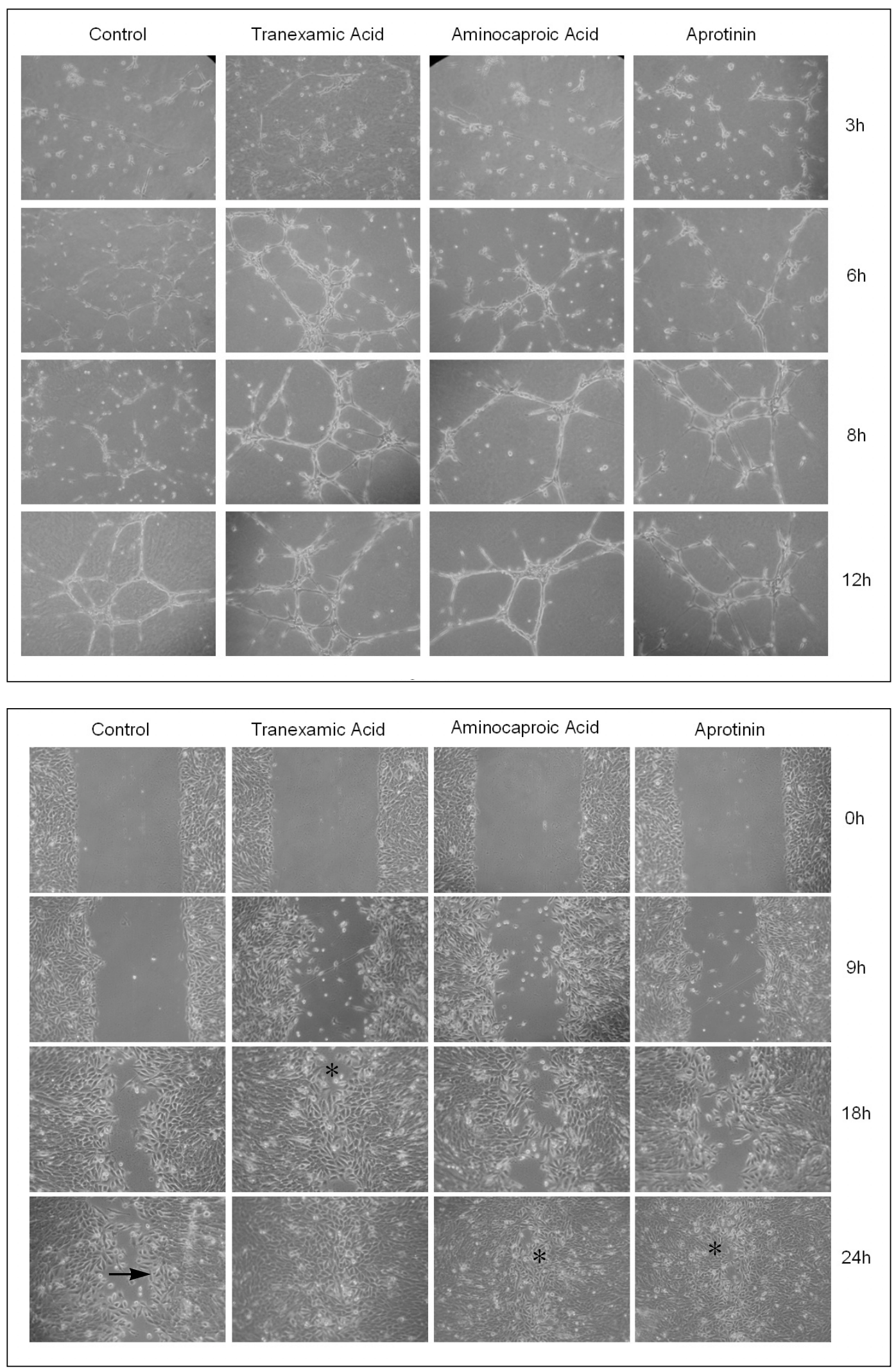

Figure 4: Effect of TA, AC and $A P$ on cord formation. Around 300,000 HMECs were seeded in P-6 matrigel-containing plates and either untreated (Control) or treated with TA (2 mM), AC

$(2 \mathrm{mM})$, or AP $(5 \mu \mathrm{g} / \mathrm{ml})$. Cells were photographed at different time points from $3 \mathrm{~h}$ to $24 \mathrm{~h}$ post-seeding.

Figure 5: Effect of TA, $A C$ and $A P$ on cell migration after wound healing. HMEC confluent monolayers were disrupted with a pipette tip, and culture medium was removed after the injury. Cells were washed in PBS, reincubated in fresh endothelial medium and either untreated (Control) or treated with TA (2 mM), AC (2 mM), or AP $(5 \mu \mathrm{g} / \mathrm{ml})$. At different time points (from 0 to $24 \mathrm{~h}$ ) cells were photographed. Asterisks mark the time points when the cells close the discontinuity. The arrow in Control after $24 \mathrm{~h}$, points to the slight discontinuity still present. antifibrinolytic agent 10 -fold more potent than aminocaproic acid and with longer half-life. Thus, it has been postulated that TA would act on HHT patients by inhibiting the fibrinolysis on the wall of telangiectases, where there is an increase of fibrino- lytic activity $(36,37)$. The only contraindication would be in those patients prone to suffer thrombosis (22).

The reports of TA performance in HHT patients are not very abundant in literature and the few known are dealing with a li- 
mited number of patients. In these sparse and limited studies, the patients showed a significant decrease in the nose bleeds with a subsequent increase in the hemoglobin levels. None of them showed side effects, nor did they need any blood transfusions. Perez-Molino (33) reported the control of a massive, life threatening haemorrhage in a HHT patient, by oral administration of TA.

The 14 patients included in the present study showed epistaxis interfering with their quality of life. All of them improved significantly, decreasing the nose bleeds and improving the levels of hemoglobin. The need for blood transfusions almost disappeared in four patients who had been depending on them before the treatment. None of the patients showed side effects derived from the TA treatment. Therefore, we can conclude that at the doses applied, up to $1 \mathrm{~g} / 8 \mathrm{~h}$, TA is a safe treatment for HHT patients.

Given the good results obtained with the TA therapy, we have explored its mechanism of action by in-vitro cellular studies, since little or nothing had been reported so far in relation to HHT. Since endoglin/ALK-1 haploinsufficiency is the general admitted origin for the HHT symptoms (38-41), TA effects on endoglin and ALK-1 were assessed. We compared TA effects with $\mathrm{AC}$, an anti-fibrinolytic less potent than TA, and with a plasmin inhibitor, AP, acting at the same level as both anti-fibrinolytic drugs. We have shown that TA improves the protein levels of endoglin and ALK-1 at the surface of the endothelial cell. AC and AP act in the same direction as TA, although AP's effect is only comparable to TA at ALK-1 protein levels, and AC is less potent increasing both endoglin and ALK-1 protein levels.

Moreover, TA also acts at the RNA level for endoglin and $A L K-1$, as proven by real-time PCR in endothelial cells. The use of $A C$ and $A P$ also shows a tendency to increase endoglin and ALK-1 RNA level, but the effect is not significant, at least after $24 \mathrm{~h}$.

Finally, the reporter experiments with constructs representing $A L K-1$ and endoglin promoters showed that TA acts at the transcriptional level, observing about a two-fold increase in both promoters after TA treatment.
In endothelial cells, TGF $\beta$ may signal through ALK-1 or ALK-5, and ALK-1 cooperates with endoglin in the transmission of the TGF $\beta$ signal (42). The use of a specific TGF- $\beta /$ ALK-1/endoglin reporter, (BRE) $)_{2}$-luc, and the corresponding ALK-5 reporter, $(\mathrm{CAGA})_{12}$-luc, showed that TA was stimulating preferentially the ALK-1/endoglin alternative, and had no effect on the ALK-5 pathway. A weak, but interesting, effect is the TA stimulation of the PAI-1 promoter in the 3TP-lux reporter. Thus, the main conclusion is that TA, in addition to the described anti-fibrinolytic effects, may trigger an increase in ALK-1 and endoglin protein levels, with the consequent stimulation of the ALK1/endoglin pathway, affected in HHT patients. This may be an additional reason why TA is more effective in HHT than in other non-HHT haemorrhages.

The way by which TA is able to stimulate this TGF $\beta$ pathway remains unclarified. Nevertheless, functional experiments like cord formation and wound healing seem to indicate that TA promotes functions dependent on the TGF $\beta /$ ALK-1/endoglin pathway $(32,34)$, since it increases the angiogenic ability and the migration rate of endothelial cells. It is worth mentioning that angiogenesis involves also migration and disruption of extracellular matrix; however, while angiogenesis and wound-healing migration is dependent on metalloproteinases (not inhibited by antifibrinolytic drugs as TA, AC or AP), fibrinolysis is caused by the serine protease plasmin (43). At this point, the fact that aprotinin mimicks the increase in migration and cord formation would mean that TA stimulates this pathway indirectly through an inhibition of plasminogen activation. This fact opens new perspectives in the search for a link between plasmin activation and the TGF $\beta$ pathway in endothelial cells.

\section{Acknowledgements}

The authors are indebted to Dr. Calvin Vary and Jonathan Berg for kindly providing the pGL2 reporter encompassing $A L K-1$ promoter This work was supported by grants from Fondo de Investigacion Sanitaria (PI020200) to CB and MEC (SAF05-01090 to LB and SAF2004-01390 to CB). A F-L is a predoctoral fellow of I3P CSIC. EG is a predoctoral fellow of MEC.

\section{References}

1. Shovlin CL, Letarte M. Hereditary haemorrhagic telangiectasia and pulmonary arteriovenous malformations: issues in clinical management and review of pathogenic mechanisms. Thorax 1999; 54: 714-729. 2. Kjeldsen AD, Vase P, Green A. Hereditary haemorrhagic telangiectasia: a population-based study of prevalence and mortality in Danish patients. J Intern Med 1999; 245: 31-39.

3. Jessurun GA, Kamphuis DJ, van der Zande FH, et al. Cerebral arteriovenous malformations in The Netherlands Antilles. High prevalence of hereditary hemorrhagic telangiectasia-related single and multiple cerebral arteriovenous malformations. Clin Neurol Neurosurg 1993; 95: 193-198.

4. Morales Angulo C, Megia Lopez R, del Valle Zapico A, et al. Rendu-Osler-Weber disease (hereditary hemorrhagic telangiectasia). Report of 30 cases. Acta Otorrinolaringol Esp 1997; 48: 625-629.

5. McAllister KA, Grogg KM, Johnson DW, et al. Endoglin, a TGF-beta binding protein of endothelial cells, is the gene for hereditary haemorrhagic telangiectasia type 1. Nat Genet 1994; 8: 345-351.
6. Johnson DW, Berg JN, Baldwin MA, et al. Mutations in the activin receptor-like kinase 1 gene in hereditary haemorrhagic telangiectasia type 2 . Nat Genet 1996; 13: 189-195.

7. Gallione CJ, Pasyk KA, Boon LM, et al. A gene for familial venous malformations maps to chromosome $9 \mathrm{p}$ in a second large kindred. J Med Genet 1995; 32: 197-199.

8. Cole SG, Begbie ME, Wallace GM, et al. A new locus for hereditary haemorrhagic telangiectasia (HHT3) maps to chromosome 5. J Med Genet 2005; 42: 577-582.

9. Plauchu H, de Chadarevian JP, Bideau A, et al. Agerelated clinical profile of hereditary hemorrhagic telangiectasia in an epidemiologically recruited population. Am J Med Genet 1989; 32: 291-297.

10. Perez del Molino A, Zarrabeitia R, Fernandez A Hereditary hemorrhagic telangiectasia. Med Clin (Barc) 2005; 124: 583-587.

11. Morales-Angulo C, del Valle-Zapico A. Hereditary hemorrhagic telangiectasia. Otolaryngol Head Neck Surg 1998; 119: 293.
12. Os AA, Friedman CM, White RI, Jr. The natural history of epistaxis in hereditary hemorrhagic telangiectasia. Laryngoscope 1991; 101: 977-980. 13. Guttmacher AE, Marchuk DA, White RI, Jr. Hereditary hemorrhagic telangiectasia. N Engl J Med 1995; 333: 918-924.

14. Morais D, Ramirez B, Santos J. Local sclerosing treatment with etoxiesclerol in ORL disease: RenduOsler disease, granuloma, angioma. Acta Otorrinolaringol Esp 2002; 53: 658-661.

15. Ramirez B, Morais D, Condado MA, et al. A new sclerosant for the treatment of Rendu-Osler-Weber disease. Acta Otorrinolaringol Esp 2000; 51: 36-39.

16. Escalante A, Pinzon A, Belloso E, et al. Cobalt therapy in the treatment of Osler-Rendu disease. Apropos of two cases. Acta Otorrinolaringol Esp 1988; 39: 57-59.

17. Urpegui AM, Abenia JM, Morandeira MJ, et al. Experimental microsurgery of the facial nerve: end-toend suture versus approximated ends wrapped with masseter muscle aponeurosis. Acta Otorrinolaringol Esp 1998; 49: 107-110. 
18. Saba HI, Morelli GA, Logrono LA. Brief report: treatment of bleeding in hereditary hemorrhagic telangiectasia with aminocaproic acid. N Engl J Med 1994; 330: 1789-1790.

19. Annichino-Bizzacchi JM, Facchini RM, Torresan $\mathrm{MZ}$, et al. Hereditary hemorrhagic telangiectasia response to aminocaproic acid treatment. Thromb Res 1999; 96: 73-76.

20. Sabba C, Gallitelli M, Palasciano G. Efficacy of unusually high doses of tranexamic acid for the treatment of epistaxis in hereditary hemorrhagic telangiectasia. N Engl J Med 2001; 345: 926

21. Klepfish A, Berrebi A, Schattner A. Intranasal tranexamic acid treatment for severe epistaxis in hereditary hemorrhagic telangiectasia. Arch Intern Med 2001; 161: 767

22. Mannucci PM. Hemostatic drugs. N Engl J Med 1998; 339: 245-253.

23. Thorsen S. Differences in the binding to fibrin of native plasminogen and plasminogen modified by proteolytic degradation. Influence of omega-aminocarboxylic acids. Biochim Biophys Acta 1975; 393: 55-65. 24. Hoylaerts M, Lijnen HR, Collen D. Studies on the mechanism of the antifibrinolytic action of tranexamic acid. Biochim Biophys Acta 1981; 673: 75-85.

25. Verstraete M. Clinical application of inhibitors of fibrinolysis. Drugs 1985; 29: 236-261.

26. Shovlin CL, Guttmacher AE, Buscarini E, et al. Diagnostic criteria for hereditary hemorrhagic telangiectasia (Rendu-Osler-Weber syndrome). Am J Med Genet 2000; 91: 66-67.

27. Sadick H, Naim R, Oulmi J, et al. Plasma surgery and topical estriol: effects on the nasal mucosa and long-term results in patients with Osler's disease. Otolaryngol Head Neck Surg 2003; 129: 233-238.

28. Fernandez-L. A, Sanz-Rodriguez F, Zarrabeitia R, et al. Blood outgrowth endothelial cells from Hereditary Haemorrhagic Telangiectasia patients reveal abnormalities compatible with vascular lesions. Cardiovasc Res 2005; 68: 235-248.

29. Sanz-Rodriguez F, Fernandez-L. A, Zarrabeitia R, et al. Mutation analysis in Spanish patients with hereditary hemorrhagic telangiectasia: Deficient endoglin up-regulation in activated monocytes. Clin Chem 2004; 50: 2003-2011.

30. Rius C, Smith JD, Almendro N, et al. Cloning of the promoter region of human endoglin, the target gene for hereditary hemorrhagic telangiectasia type 1 . Blood 1998; 92: 4677-4690.

31. Botella LM, Sanchez-Elsner T, Rius C, et al. Identification of a critical $\mathrm{Sp} 1$ site within the endoglin promoter and its involvement in the transforming growth factor-beta stimulation. J Biol Chem 2001; 276: 34486-34494.

32. Blanco FJ, Santibanez JF, Guerrero-Esteo M, et al. Interaction and functional interplay between endoglin and ALK-1, two components of the endothelial transforming growth factor-beta receptor complex. J Cell Physiol 2005; 204: 574-584.

33. Perez del Molino A, Zarrabeitia R, Fernandez A, et al. Efficacy of tranexamic acid in a patient with hereditary hemorrhagic telangiectasia and massive epistaxis. Med Clin (Barc) 2004; 123: 118-119.

34. Lebrin F GM, Jonker L, Carvalho RL, et al. Endoglin promotes endothelial cell proliferation and TGF-beta ALK-1 signal transduction. EMBO J 2004; 23: 4018-4028. 35. Byahatti SV, Rebeiz EE, Shapshay SM. Hereditary hemorrhagic telangiectasia: what the otolaryngologis should know. Am J Rhinol 1997; 11: 55-62.

36. Kwaan HC, Silverman S. Fibrinolytic activity in lesions of hereditary hemorrhagic telangiectasia. Arch Dermatol 1973; 107: 571-573.

37. Watanabe M, Hanawa S, Morishima T. Fibrinolytic activity in cutaneous lesions of hereditary hemorrhagic telangiectasia. Nippon Hifuka Gakkai Zasshi 1985; 95 : $11-16$

38. Shovlin CL, Hughes JM, Scott J, et al. Characterization of endoglin and identification of novel mutations in hereditary hemorrhagic telangiectasia. Am J Hum Genet 1997; 61: 68-79.

39. Gallione CJ, Klaus DJ, Yeh EY, et al. Mutation and expression analysis of the endoglin gene in hereditary hemorrhagic telangiectasia reveals null alleles. Hum Mutat 1998; 11: 286-294.

40. Abdalla SA, Geisthoff UW, Bonneau D, et al. Visceral manifestations in hereditary haemorrhagic telangiectasia type 2. J Med Genet 2003; 40: 494-502.

41. Abdalla SA, Letarte M. Hereditary haemorrhagic telangiectasia: current views on genetics and mechanisms of disease. J Med Genet 2006; 43: 97-110.

42. Lebrin F, Deckers M, Bertolino P, et al. TGF-beta receptor function in the endothelium. Cardiovasc Res 2005; 65: 599-608

43. Laurens N, Koolwijk P, de Maat MP. Fibrin structure and wound healing. J Thromb Haemost 2006; 4 : 932-939. Review.

\section{Yearbook of Medical Informatics complete your collection?}

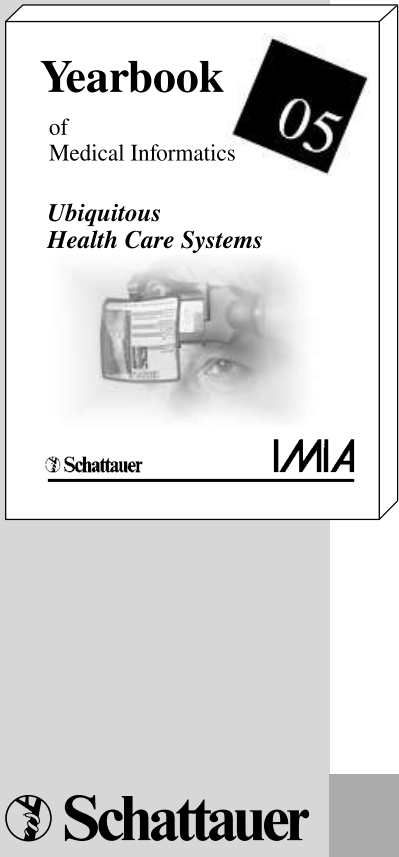

Haux/Kulikowski (Eds.)

Yearbook of Medical Informatics 2005

2005. 592 pages, paperback $€ 95.00^{*}(\mathrm{D}) / € 97.70^{*}(\mathrm{~A}) / \mathrm{CHF} 152.00^{*}$

Students:

$€ 24.75^{*}(\mathrm{D}) / € 25.50^{*}(\mathrm{~A}) / \mathrm{CHF} 40.00^{*}$

Special offer for Members of IMIA Society:

$€ 36.00^{*}(\mathrm{D}) / € 37.00^{*}(\mathrm{~A}) / \mathrm{CHF} 58.00^{*}$

ISBN 978-3-7945-2397-9

*Mailing costs (surface) included
The special research section in 2005: Ubiquitous Health Care Systems

Information technology helps to improve the quality of health care by disseminating and systematizing knowledge of diagnostic and therapeutic possibilities as well as the organization and management of care.

Unobtrusive, active, non-invasive technologies, including wearable devices, allow us to continuously monitor and respond to changes in the health of a patient. Such devices range from micro-sensors, integrated in textiles, through consumer electronics, embedded in fashionable clothes, to beltworn personal computers with head mounted displays. Such ubiquitous computing allows us to identify new ways of managing care that promises to be considerably easier in letting patients maintain their good health while enjoying their life in their usual social settings, rather than having to spend much time at costly, dedicated healthcare facilities. It may prove essential for ensuring quality of life as well as health care for increasingly aging societies.

The section comprises a selection of some of the most advanced research publications in this challenging field

The IMIA Yearbook of Medical Informatics also presents original reviews by senior scientists describing the current developments of Health and Medical Informatics and keeps its reader informed about international education programs of the field. The Yearbook of Medical Informatics provides information about the work of IMIA and IMIA regional groups. 